\title{
One-dimensional half-metallic interfaces of two-dimensional honeycomb insulators
}

\author{
N. C. Bristowe, ${ }^{1,2}$ Massimiliano Stengel, ${ }^{3,4}$ P. B. Littlewood, ${ }^{1,5}$ Emilio Artacho, ${ }^{1,6,7}$ and J. M. Pruneda ${ }^{8,9}$ \\ ${ }^{1}$ Theory of Condensed Matter, Cavendish Laboratory, University of Cambridge, Cambridge CB3 OHE, United Kingdom \\ ${ }^{2}$ Theoretical Materials Physics, University of Liège, B-4000 Sart-Tilman, Belgium \\ ${ }^{3}$ Institució Catalana de Recerca i Estudis Avançats, 08010 Barcelona, Spain \\ ${ }^{4}$ Institut de Ciència de Materials de Barcelona, CSIC, Campus UAB, 08193 Bellaterra, Spain \\ ${ }^{5}$ Physical Sciences and Engineering, Argonne National Laboratory, Argonne, Illinois 60439, USA \\ ${ }^{6}$ CIC Nanogune and DIPC, Tolosa Hiribidea 76, 20018 San Sebastian, Spain \\ ${ }^{7}$ Basque Foundation for Science Ikerbasque, 48011 Bilbao, Spain \\ ${ }^{8}$ ICN2 - Institut Catala de Nanociencia i Nanotecnologia, Campus UAB, 08193 Bellaterra (Barcelona), Spain \\ ${ }^{9}$ CSIC - Consejo Superior de Investigaciones Cientificas, ICN2 Building, Campus UAB ,08193 Bellaterra (Barcelona), Spain
}

(Received 8 October 2012; published 30 October 2013)

\begin{abstract}
We study zigzag interfaces between insulating compounds that are isostructural to graphene, specifically II-VI, III-V, and IV-IV two-dimensional honeycomb insulators. We show that these one-dimensional interfaces are polar, with a net density of excess charge that can be simply determined by using the ideal (integer) formal valence charges, regardless of the predominant covalent character of the bonding in these materials. We justify this finding on fundamental physical grounds by analyzing the topology of the formal polarization lattice in the parent bulk materials. First-principles calculations elucidate an electronic compensation mechanism not dissimilar to oxide interfaces, which is triggered by a Zener-like charge transfer between interfaces of opposite polarity. In particular, we predict the emergence of one-dimensional electron and hole gases, which in some cases are ferromagnetic half metallic.
\end{abstract}

DOI: 10.1103/PhysRevB.88.161411

PACS number(s): 73.22.-f, 73.20.-r, 73.21.-b, 75.70.-i

Since the first two-dimensional material (graphene) was successfully synthesized by mechanical exfoliation, ${ }^{1}$ a growing number of studies have been devoted to other planar systems. These include BN monolayers, ${ }^{2}$ layered transition metal oxides, ${ }^{3}$ dichalcogenides, ${ }^{4,5}$ and topological insulators such as $\mathrm{Bi}_{2} \mathrm{Te}_{3}$ or $\mathrm{Bi}_{2} \mathrm{Se}_{3} .{ }^{6}$ Of particular note in this context are a number of II-VI, III-V, and IV-IV compounds, whose stable bulk phase is wurtzite but which may adopt a planar graphitic phase in ultrathin films. These were first predicted theoretically several years ago ${ }^{7,8}$ and were later grown in the laboratory.., 10

Recent improvements in growth techniques allow joining these nanosheets together. In addition to the many heterostructures studied so far involving graphene and $\mathrm{BN}$, obtained by modified stacking ${ }^{11}$ or segregated nanosheets, ${ }^{12,13}$ there are and will be coplanar heterostructures, ${ }^{14,15}$ made from different insulators on the same sheet, with many of them having a significant degree of ionic character. This brings up the important question of whether "polar discontinuities" 16 might play a role in these low-dimensional systems. Vertical stacking of nanosheets is not dissimilar to oxide interfaces, ${ }^{17}$ and polarity effects will resemble those of ultrathin films already discussed in the literature. ${ }^{7,8}$ However, to our knowledge, coplanar interfaces between two-dimensional (2D) insulators have not been addressed from first principles, except for a qualitative discussion of the electrostatics. ${ }^{18}$

Compared with the case of polar oxide interfaces, there are a few additional challenges that are specific to the $2 \mathrm{D}$ case. First, the electrostatics is somewhat more complicated: unlike a plane of charge [interface between three-dimensional (3D) materials], which generates a uniform electric field in the whole space [essentially a one-dimensional (1D) problem], a line of charge (interface between $2 \mathrm{D}$ materials) produces a logarithmically divergent potential with inhomogeneous stray fields in the vacuum region. Second, the symmetry group (with a main sixfold rotation axis but no center of symmetry) gives rise to different polarization classes than the ones found in centrosymmetric insulators. ${ }^{19}$ This calls for special care in the application of the modern theory of polarization, particularly of the interface theorem ${ }^{20}$ for the determination of the excess "bound" charge at a given interface. Moreover, the lack of center of symmetry implies that these materials are piezoelectrically active.

Here we perform extensive density-functional theory calculations to show that (i) there indeed exists a net charge at the 1D zigzag interface between two chemically distinct polar insulating honeycomb domains, (ii) the linear charge density is uniquely determined from bulk properties of the participating materials and is related to the nontrivial topology of their respective polarization lattice, (iii) the divergent electrostatic potential produced by the lines of charge triggers a Zener-like breakdown mechanism in the limit of increasingly wide domains, and (iv) such a mechanism gives rise to spin-polarized one-dimensional electron/hole gases (1DEG/1DHG). Notice that points (i) and (ii) are completely general and are applicable to any 1D interface in 2D materials, including grain boundaries. In what follows, we proceed by addressing points (i) to (iv) in the same sequential order.

According to the modern theory of polarization ${ }^{20}$ and as further elucidated in Refs. 21-23, the net charge at the interface between two compounds can be exactly given by invoking only bulk properties of the parent materials. In particular, one needs to consider the lattice (Fig. 1) of allowed values for the socalled formal polarization $\mathbf{P}$ of either crystalline constituent. Then, based on the interface orientation and termination, ${ }^{23}$ one can readily deduce the interface net charge. An intuitive 
(a)

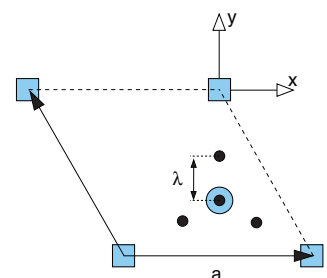

(b)

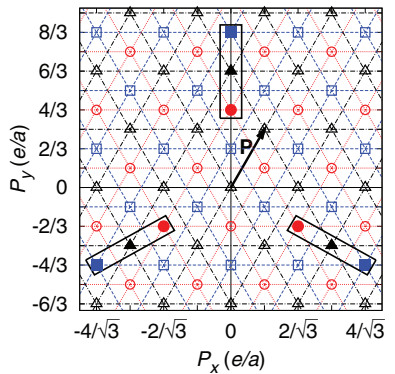

(c)

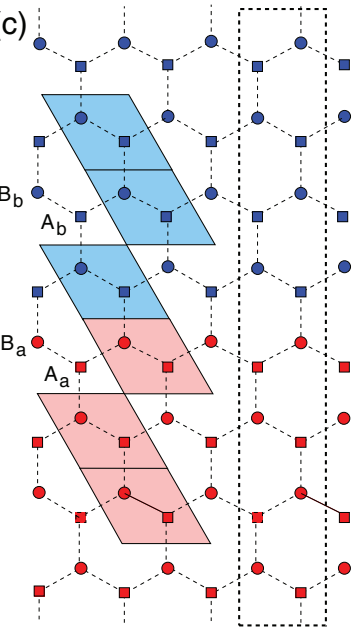

FIG. 1. (Color online) (a) The position of the Wannier centers (small circles) in the primitive unit cell of $\mathrm{ZnO}, \mathrm{AlN}$, and $\mathrm{SiC}$. The "cation" $(\mathrm{Zn}, \mathrm{Al}, \mathrm{Si})$ is represented by squares, and the "anion" $(\mathrm{O}, \mathrm{N}, \mathrm{C})$ is represented by circles. Here $a$ is the cell parameter (3.316, 3.121, and $3.110 \AA$ for $\mathrm{ZnO}, \mathrm{AlN}$, and $\mathrm{SiC}$, respectively), and $\lambda$ is the distance of the $s p^{2}$ Wannier centers from the anion ( $\lambda=0.431,0.491,0.677 \AA$, respectively), which increases with the bond covalency. (b) The triangular lattice of allowed polarization values for the II-VI (red circles), III-V (black triangles), and IV-IV (blue squares) 2D honeycomb compounds. Each symbol represents a polarization vector, indicated by $P$. The box highlights the values obtained with "Wannier anions" (see text). (c) Sketch of the zigzag interface between two compounds $\left(\mathrm{A}_{a} \mathrm{~B}_{a}\right.$ in red and $\mathrm{A}_{b} \mathrm{~B}_{b}$ in blue) with an example simulation box indicated by dashed lines.

way to do this is by representing the electronic structure of the material in terms of localized Wannier functions. ${ }^{24-26}$ For polarization purposes, these effectively map the continuous charge density distribution of the periodic crystal into a discrete set of classical point charges, located at the Wannier centers ${ }^{27}$ shown in Fig. 1(a). Then the formal polarization of the crystal is simply given by the total dipole moment of the ensemble of these charges and the ion cores divided by the unit-cell volume. Of course, there are infinite possible choices for the primitive basis of atoms and Wannier orbitals, hence the multivaluedness of the formal $\mathbf{P}$ [see Fig. 1(b)].

A particularly convenient approach is that of combining the valence Wannier functions with their nearest-neighbor ion core, obtaining a negatively charged object that we call a "Wannier anion." 23 This way, we obtain polarization lattices that include only a subset of the original points [larger symbols in Fig. 1(b)]. More specifically, each of these restricted points simply corresponds to the dipole moment of a system of two integer point charges $Q= \pm N e$, placed at two arbitrarily chosen lattice sites, where $N$ is the "formal valence" of the cation in each compound. Note that, due to the threefold symmetry of these structures, both the Wannier anion and the cation carry zero dipole moment; hence, the formal polarization only contains the point-charge contribution. ${ }^{23}$ This has some analogies with the $\mathrm{LaAlO}_{3} / \mathrm{SrTiO}_{3}$ case, where it was shown that covalency effects are also irrelevant to charge-counting purposes. ${ }^{23,28}$

Each arbitrary choice of atomic basis, in turn, yields a welldefined interface termination once the unit cell is periodically repeated to generate a semi-infinite domain. For the zigzag interfaces considered in this work, the appropriate choice of the bulk unit cell (i.e., one of the many choices that tile the whole system without leaving any unpaired ion at the interface) is that shown by shaded regions in Fig. 1(c). The dipole moment along $y$ of such a unit cell is given by $d=-Q a /(2 \sqrt{3})$, yielding a formal polarization of $P_{y}=-Q / 3 a$. The interface theorem relates differences in formal polarization to the net bound charge at the interface, and we have

$$
\sigma_{\text {bound }}=P_{y}^{(b)}-P_{y}^{(a)}=-\frac{\Delta Q}{3 a} .
$$

This implicitly assumes that both parent compounds are hexagonal and have the same equilibrium lattice parameter. In practice there is always a small lattice mismatch, and to realize a pseudomorphic interface one or both constituents need to be strained. Then, Eq. (1) must be corrected by piezoelectric contributions on $P_{y}$.

The electrostatic potential produced by this interfacial net charge diverges in the limit of increasingly wide domains and needs to be somehow neutralized. To investigate a possible compensation mechanism, we use explicit first-principles calculations of 2D superlattices, which are constructed by periodically repeating two compositionally distinct nanoribbons along the stacking direction $(y)$, so that the interface direction $(x)$ follows the zigzag edge of the hexagonal lattice [see Fig. 1(c)], consistent with the above discussion of the bulk polarity. Similar heterostructures made from $\mathrm{C}$ and $\mathrm{BN}$ domains have been discussed before in the literature. ${ }^{30,38} \mathrm{We}$ chose $\mathrm{SiC}, \mathrm{AlN}$, and $\mathrm{ZnO}$ as representative compounds for groups IV-IV, III-V, and II-VI, respectively. The periodicity of the superlattices is controlled by varying the nanoribbon width $m$ (given by the number of AB zigzag chains). Only stoichiometric compounds are considered, which means that two chemically distinct interfaces are present in our superlattices: one of type $\mathrm{A}_{a} \mathrm{~B}_{a} \mathrm{~A}_{b} \mathrm{~B}_{b}\left(\mathrm{~A}_{a}\right.$ : cation of material $a$, $\mathrm{B}_{b}$ : anion of material $b$, etc.) and one of type $\mathrm{A}_{b} \mathrm{~B}_{b} \mathrm{~A}_{a} \mathrm{~B}_{a}$. Along the normal direction to the sheet we include a vacuum layer of $20 \AA$, sufficiently thick to obtain converged results (no significant differences are observed with a vacuum layer of up to $200 \AA$ ). The calculations are performed using the spin-polarized Perdew-Burke-Ernzerhof (PBE) exchange correlation functional ${ }^{31}$ as implemented in the SIESTA code. ${ }^{32,33}$ Norm-conserving pseudopotentials ${ }^{34}$ and a double- $\zeta$ polarized basis set are used to represent the valence electrons. ${ }^{35}$ Atomic forces are relaxed to less than $20 \mathrm{meV} / \AA$.

Figure 2(a) shows the spin-resolved layer-by-layer density of states (DOS) of the AlN-SiC $(m=16)$ superlattice (IIIV/IV-IV). The presence of a macroscopic sawtooth-like potential, corresponding to electric fields of opposite polarity in the two material regions, is clearly visible from the plot. These fields are generated by the net interfacial charges discussed above. Under this potential generated by the polarization discontinuity and for increasing thickness, the valence band maximum (VBM) at the $p$-type (Al-C) interface eventually becomes higher in energy than the conduction band minimum (CBM) at the $n$-type ( $\mathrm{Si}-\mathrm{N})$ interface, and a charge transfer becomes favorable in energy. The resulting Zener charge 


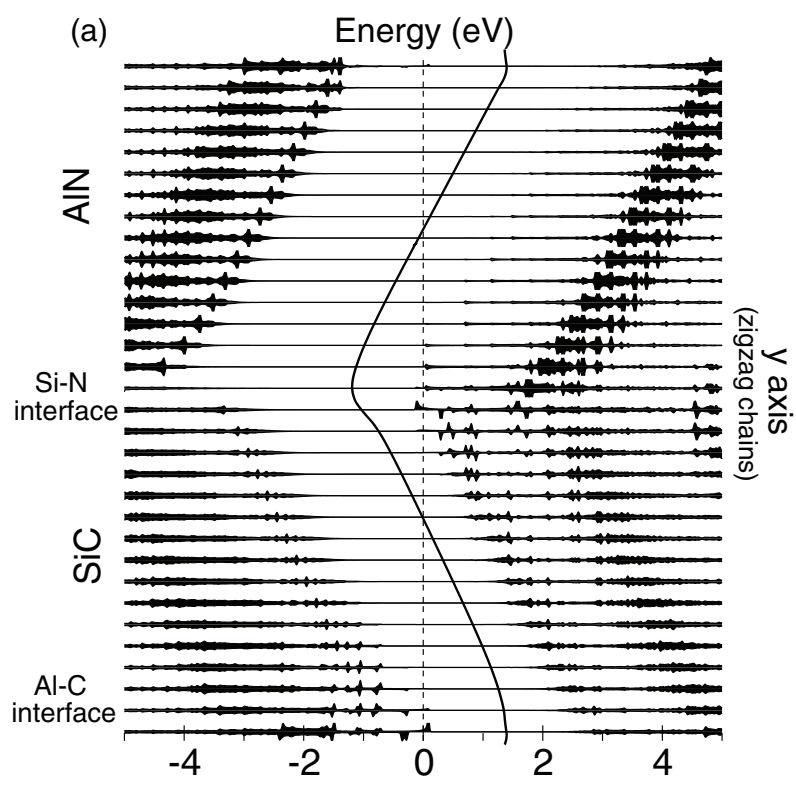

(b)

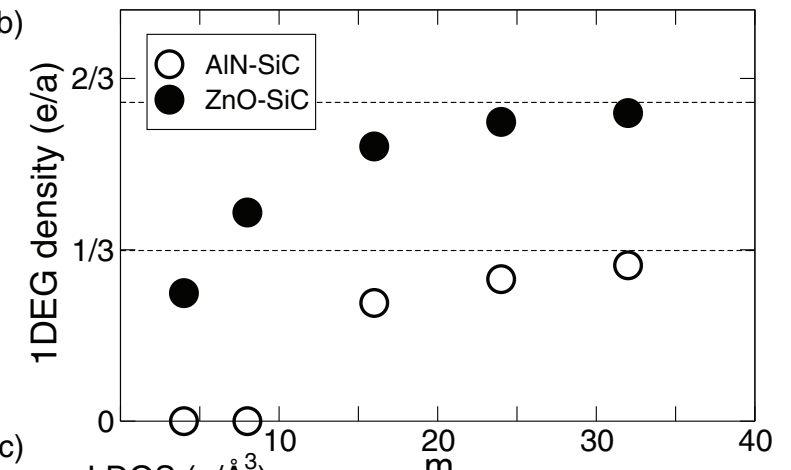

(c)
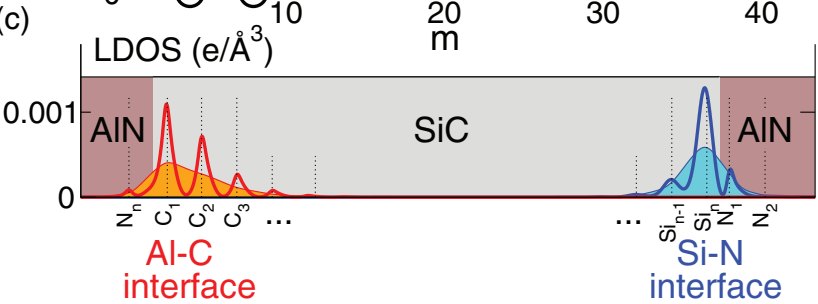

FIG. 2. (Color online) (a) Spin-resolved layer-by-layer density of states of the AlN-SiC $(m=16)$ superlattice. The top half corresponds to the AlN slab, and the bottom half corresponds to the SiC slab, with the interface terminations indicated. The solid black line shows the macroscopically averaged electrostatic potential. (b) The density of free electrons (or holes) calculated as a function of slab width $m$ for the two superlattice systems studied (AlN-SiC and $\mathrm{ZnO}-\mathrm{SiC}$ ). Theoretical values corrected by piezoelectric effects are marked by dashed lines. (c) The spatial (plane-averaged) charge distribution computed from the local density of states (LDOS; shaded curves are macroscopically averaged LDOS).

transfer gives rise to an accumulation of free carriers along the interfaces which produce the aforementioned 1DEG/1DHG, whose spatial localization can be appreciated from their calculated planar-averaged densities, plotted in Fig. 2(b). The $1 \mathrm{DEG} / 1 \mathrm{DHG}$, in turn, partially neutralize the net charge at either interface, reducing the potential offset. This feedback mechanism results in an effective pinning of the $p \mathrm{VBM}$ and the $n$ CBM, which remain close in energy regardless of the value of $m$ after breakdown occurs. Thus, many aspects of the present electronic mechanism resemble those already reported for oxide superlattices. ${ }^{28}$ However, we stress that the electrostatics of this system is different and analogous to that of an infinite array of alternating positive and negative linear charge densities.

At smaller ribbon widths (not shown) the associated potential drop is smaller than the electronic band gap of either participating compound, and the system can remain insulating (i.e., no "Zener breakdown" occurs). In the large- $m$ limit, on the other hand, the 1DEG/1DHG densities tend to the "ideal" value, which corresponds to perfectly neutralizing the discontinuity in the normal component of the bulk $\mathbf{P}$, according to Eq. (1). This dependence of the charge transfer with ribbon width is shown in Fig. 2(c) for both AlN-SiC (III-V/IV-IV) and ZnO-SiC (II-VI/IV-IV) interfaces. This nicely shows a crossover scenario from a short-period uncompensated regime to a longer-period regime where a Zener-like breakdown mechanism occurs, similar to the case of a $\mathrm{LaAlO}_{3}-\mathrm{SrTiO}_{3}$ superlattice. The precise details of the crossover depends on the band gaps and alignments between the two materials, which might not be properly given by density functional theory calculations,${ }^{30}$ although the physical mechanism remains valid. Note that the asymptotic limits [dashed lines in Fig. 2(c)], in fact, do not correspond exactly to the ideal rational values $(1 / 3$ and $2 / 3)$ of Eq. (1). This is due to piezoelectric effects, which again are accounted for by macroscopic bulk properties of the participating materials. In particular, the lattice mismatch between $\mathrm{SiC}$ and $\mathrm{ZnO}$ forces a compression in the $\mathrm{ZnO}$ ribbon (and a minor expansion in $\mathrm{SiC}$ ), resulting in a shift of its formal polarization lattice along the axis normal to the interface, hence decreasing $P_{\mathrm{SiC}}-P_{\mathrm{ZnO}}$.

We find that the 1D gases of carriers at the interfaces of these insulating materials are fully spin polarized. To further investigate magnetic effects, we plot in Fig. 3 the band structure near the Fermi level $E_{f}$ for a AlN-SiC superlattice with a thickness that corresponds to the uncompensated regime [Fig. 3(a)] and a partially compensated superlattice with a larger thickness [Figs. 3(b)-3(b)]. There are three dispersive $\pi$ bands close to $E_{f}$ that are involved in the compensation

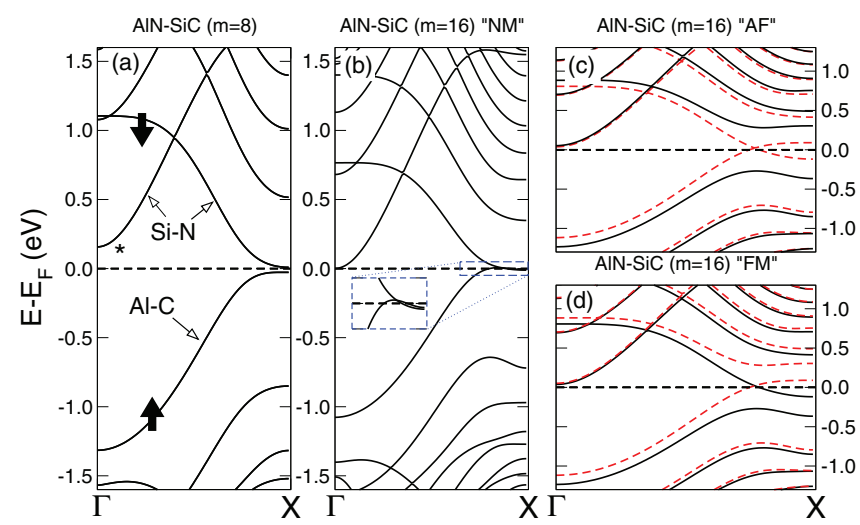

FIG. 3. (Color online) The band structure of the AlN-SiC superlattices for (a) $m=8$ and $m=16$ with (b) paramagnetic (NM), (c) antiferromagnetic (AF), and (d) ferromagnetic (FM) solutions. Solid black and dashed red lines correspond to bands with different spin components. The inset in (b) shows the Fermi-level crossing of bands at the zone boundary. 
mechanism, one occupied and mostly localized at the Al-C interface and two empty and mostly localized at the Si-N interface. The flat bands at $X$ for the nonmagnetic solutions [Figs. 3(a) and 3(b)] very much resemble the edge states in graphene nanoribbons described with a simple tight-binding model for the $\pi$ electrons. ${ }^{36}$ The corresponding high density of electronic states at $E_{f}$ suggests magnetic ordering due to a Stoner instability.

Indeed, two spin-polarized phases with ferromagnetic orderings along the interface are lower in energy than the paramagnetic solution and correspond to the transfer of charge (electrons) with well-defined spin from one interface ( $p \mathrm{VBM}$ ) to the other $(n \mathrm{CBM})$ : when the spin is preserved in the transfer, as in Fig. 3(c), the electronic magnetization at both interfaces is antiferromagnetically aligned (AF), whereas a spin flip of the transferred charge gives the ferromagnetic (FM) solution [Fig. 3(d)]. The two solutions, AF and FM between interfaces, are almost degenerate in energy because the 1DEG and the $1 \mathrm{DHG}$ are decoupled by the wide insulating ribbon. Notice that the whole system is half metallic for the AF solution, with full spin polarization of the conducting electrons.

It is generally agreed that a one-dimensional metal is unstable with respect to a symmetry-lowering modulation of the charge. ${ }^{37}$ The coupling between lattice vibrations and electrons near the Fermi level drives Peierls distortions that open a gap. Such a deformation could also be a way to resolve the instability due to the large density of states at the Fermi level shown in Fig. 3(b). To address the stability of the 1DEG against Peierls distortions, calculations using zone-folding techniques were performed for the AlN-SiC $(m=16)$ superlattice, including up to three or four unit cells along the $x$ axis (in the limit of large $m$ a supercell with three unit cells should be enough to accommodate symmetry-lowering deformations capable of opening a gap in the right region of the Brillouin zone). We did not detect any trace of bond-length alternations in our tests, all of which remained in a metallic state. This is in agreement with previous studies that showed that Peierls distortions can open a gap in similar edge bands only for narrow ( $<6 \AA$ ) graphene ${ }^{38}$ and graphane nanoribbons. ${ }^{39}$
In addition to competing structural/electronic ground states, another factor that could thwart experimental realization of the 1DEG/1DHG predicted here is charged defects, similar to those discussed in $\mathrm{C} / \mathrm{BN}$ superlattices. ${ }^{29}$ Nevertheless, the defect concentration that would be required is of the scale of one every few interface atoms, which may be thermodynamically stable, but its appearance would crucially depend on the kinetics of its formation. Although the study of these processes is beyond the scope of this Rapid Communication, we simply notice here that the growth of the pristine interface vs charged chemical defects will depend on the growth conditions and procedure, and it is likely possible to favor one over the other by tuning growth parameters. The typical ppm scale of defects would have hardly any effect in this sense. Low concentrations of defects might, however, modify the properties of the 1DEGs and enable engineering of the electronic properties of the 1DEGs for potential applications in spintronics, sensing, and electronics.

In conclusion, we have shown that polar compensation mechanisms can give rise to fully spin-polarized onedimensional electron and hole gases at the interface between two coplanar insulating bidimensional materials, in close analogy with the $3 \mathrm{D}$ case of the $\mathrm{LaAlO}_{3} / \mathrm{SrTiO}_{3}(001)$ interface. The net charge at the interface can be determined solely from bulk properties (the polarization) of the parents' materials, as required by the interface theorem. ${ }^{20}$ The polarization is given by a simple charge-counting procedure, irrespective of covalency/ionicity, that results in three distinct sets of allowed polarization lattices for the II-VI, III-V, and IV-IV compounds and in the formation of new electronic states (1DEGs) at the interface between two domains corresponding to different polarization classes.

We acknowledge I. Souza for valuable discussions, the support of EPSRC, MCINN (Grants No. FIS2009-12721-C0401 and No. CSD2007-00041), and computing resources of CamGRID and Darwin at Cambridge, the Spanish Supercomputer Network, and HPC Europa. P.B.L. acknowledges DOE support under Grant No. FWP 70069.
${ }^{1}$ K. Novoselov, A. Geim, S. Morozov, D. Jiang, Y. Zhang, S. Dubonos, I. Grigorieva, and A. Firsov, Science 306, 666 (2004).

${ }^{2}$ D. Pacilé, J. C. Meyer, C. O. Girit, and A. Zettl, Appl. Phys. Lett. 92, 133107 (2008).

${ }^{3}$ M. Osada and T. Sasaki, J. Mater. Chem. 19, 2503 (2009).

${ }^{4}$ C. Lee, H. Yan, L. E. Brus, T. F. Heinz, J. Hone, and S. Ryu, ACS Nano 4, 2695 (2010).

${ }^{5}$ J. C. Coleman, M. Lotya, A. O’Neill, S. D. Bergin, P. J. King, U. Khan, K. Young, A. Gaucher, S. De, R. J. Smith et al., Science 331, 568 (2011)

${ }^{6}$ D. Teweldebrhan, V. Goyal, M. Rahman, and A. A. Balandin, Appl. Phys. Lett. 96, 053107 (2010).

${ }^{7}$ J. Goniakowski, C. Noguera, and L. Giordano, Phys. Rev. Lett. 93, 215702 (2004).

${ }^{8}$ C. L. Freeman, F. Claeyssens, N. L. Allan, and J. H. Harding, Phys. Rev. Lett. 96, 066102 (2006).
${ }^{9}$ C. Tusche, H. L. Meyerheim, and J. Kirschner, Phys. Rev. Lett. 99, 026102 (2007).

${ }^{10}$ S. S. Lin, J. Phys. Chem. C 116, 3951 (2012).

${ }^{11}$ C. R. Dean, A. F. Young, I. Meric, C. Lee, L. Wang, S. Sorgenfrei, K. Watanabe, T. Taniguchi, P. Kim, K. L. Shepard et al., Nat. Nanotechnol. 5, 722 (2010).

${ }^{12}$ L. Ci, L. Song, C. Jin, D. Jariwala, D. Wu, Y. Li, A. Srivastava, Z. F. Wang, K. Storr, L. Balicas et al., Nat. Mater. 9, 430 (2010).

${ }^{13}$ M. P. Levendorf, C.-J. Kim, L. Brown, P. Y. Huang, R. W. Havener, D. A. Muller, and J. Park, Nature (London) 488, 627 (2012).

${ }^{14}$ M. Cañas-Ventura, W. Xiao, D. Wasserfallen, K. Müllen, H. Brune, J. Barth, and R. Fasel, Angew. Chem., Int. Ed. 46, 1814 (2007).

${ }^{15}$ H. Şahin, S. Cahangirov, M. Topsakal, E. Bekaroglu, E. Akturk,

R. T. Senger, and S. Ciraci, Phys. Rev. B 80, 155453 (2009).

${ }^{16}$ A. Ohtomo and H. Y. Hwang, Nature (London) 427, 423 (2004).

${ }^{17}$ J. Mannhart and D. Schlom, Science 327, 1607 (2010). 
${ }^{18}$ J. Goniakowski and C. Noguera, Phys. Rev. B 83, 115413 (2011).

${ }^{19}$ P. Jadaun, D. Xiao, Q. Niu, and S. K. Banerjee, Phys. Rev. B 88, 085110 (2013).

${ }^{20}$ D. Vanderbilt and R. D. King-Smith, Phys. Rev. B 48, 4442 (1993).

${ }^{21}$ M. Stengel and D. Vanderbilt, Phys. Rev. B 80, 241103 (2009).

${ }^{22}$ N. Bristowe, P. Littlewood, and E. Artacho, J. Phys. Condens. Matter 23, 081001 (2011).

${ }^{23}$ M. Stengel, Phys. Rev. B 84, 205432 (2011).

${ }^{24}$ N. Marzari and D. Vanderbilt, Phys. Rev. B 56, 12847 (1997).

${ }^{25}$ M. Stengel and N. A. Spaldin, Phys. Rev. B 73, 075121 (2006).

${ }^{26}$ N. Marzari, A. A. Mostofi, J. R. Yates, I. Souza, and D. Vanderbilt, Rev. Mod. Phys. 84, 1419 (2012).

${ }^{27}$ The Wannier calculation was performed with the WANNIER90 (Ref. 41) code and the density functional theory code SIESTA (Refs. 32 and 33).

${ }^{28}$ N. C. Bristowe, E. Artacho, and P. B. Littlewood, Phys. Rev. B 80, 045425 (2009).

${ }^{29}$ J. M. Pruneda, Phys. Rev. B 85, 045422 (2012).

${ }^{30}$ M. Bernardi, M. Palummo, and J. C. Grossman, Phys. Rev. Lett. 108, 226805 (2012).
${ }^{31}$ J. P. Perdew, K. Burke, and M. Ernzerhof, Phys. Rev. Lett. 77, 3865 (1996).

${ }^{32}$ P. Ordejon, E. Artacho, and J. M. Soler, Phys. Rev. B 53, 10441 (1996).

${ }^{33}$ J. Soler, E. Artacho, J. Gale, A. Garcia, J. Junquera, P. Ordejon, and D. Sanchez-Portal, J. Phys. Condens. Matter 14, 2745 (2002).

${ }^{34}$ N. Troullier and J. L. Martins, Phys. Rev. B 43, 1993 (1991).

${ }^{35}$ E. Anglada, J. M. Soler, J. Junquera, and E. Artacho, Phys. Rev. B 66, 205101 (2002).

${ }^{36}$ M. Fujita, K. Wakabayashi, K. Nakada, and K. Kusakabe, J. Phys. Soc. Jpn. 65, 1920 (1996).

${ }^{37}$ R. E. Peierls, Quantum Theory of Solids (Clarendon, Oxford, 1955).

${ }^{38}$ L. Pisani, J. A. Chan, B. Montanari, and N. M. Harrison, Phys. Rev. B 75, 064418 (2007).

${ }^{39}$ V. Tozzini and V. Pellegrini, Phys. Rev. B 81, 113404 (2010).

${ }^{40}$ J. M. Pruneda, Phys. Rev. B 81, 161409(R) (2010).

${ }^{41}$ A. Mostofi, J. Yates, Y. Lee, I. Souza, D. Vanderbilt, and N. Marzari, Comput. Phys. Commun. 178, 685 (2008). 